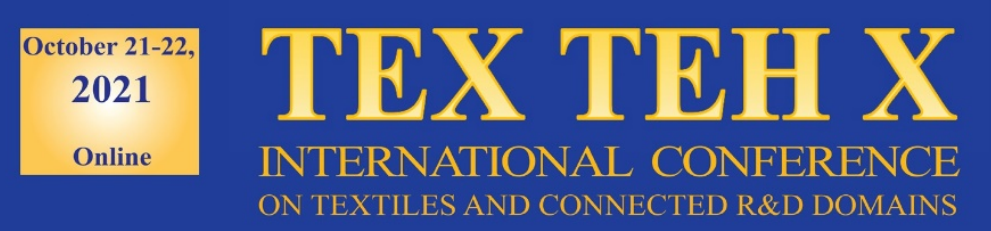

\title{
ANTIMICROBIAL ACTIVITY OF FIR FUNCTIONALIZED TEXTILE MATERIALS
}

DOI: 10.35530/TT.2021.57

\author{
O. Iordache, E.C. Tanasescu, I. Sandulache, C. Lite, L.O. Secareanu, \\ E. Perdum
}

National Research Development Institute for Textile and Leather, Romania

(E-mail: iordacheovidiu.g@gmail.com)

\begin{abstract}
Far Infrared (FIR) functionalized textile materials are enjoying a special attention nowadays, as a viable and practical solution for treating a wide range of medical conditions (relief of acute or chronic inflammation and circulatory problems, prevention of microbial infections, improvement of nervous system functions, reduction of skin lipids, improvement of blood circulation, removal of accumulated toxins by improving lymphatic circulation etc.). At the molecular level, FIR compounds and functionalized materials exert strong rotational and vibrational effects, with beneficial biological potential. These materials are based on the principle of absorbing light energy and then irradiating this energy back into the body at specific wavelengths. FIR functionalized textile materials are a new category of functional textiles that have the potential to improve well-being and health. Present paper explored the antimicrobial potential of four textile materials, functionalized with FIR, UV protection and antimicrobial functionalization compounds, tested according to two methods for assessment of antimicrobial character: a testing method in dynamic conditions and a testing method in static conditions. The evaluation of the antimicrobial character showed very good rates of reduction of the microbial population, of the functionalized textile materials, following the testing on four strains of pathogenic fungi: Candida albicans, Epydermophyton floccosum, Tricophyton interdigitale and Aspergillus niger, with reduction rates between $76.16 \%$ and $96.06 \%$.
\end{abstract}

Keywords: FIR, far infrared, textiles, antimicrobial

\section{INTRODUCTION}

Pyroelectric materials are functional materials that can generate an electrical response following a change in temperature. Modern solutions often include a combination of polypropylene and special lead-free bio-ceramics to create functional FI garments that are materialized in commercially available products such as socks, pillows, linens, knee pads, pants, bedspreads, bed linen, shoulder pads etc. [1]. Several studies have suggested improvement in patients' condition that suffered of atopic dermatitis in response to the use of special fabrics in the affected area.

Previous studies have also shown that the effects of infrared radiation can activate fibroblasts, increase collagen synthesis and the expression of growth factor-beta1 (TGFbeta1) in rat wounds. Thus, particles of germanium $(\mathrm{Ge})$ and silicon dioxide $\left(\mathrm{SiO}_{2}\right)$ were incorporated at the nano scale into polyvinyl alcohol (PVA) nanofibers. The emission wavelength of these nanofiber membranes was between $5-20 \mu \mathrm{m}$ at $37^{\circ} \mathrm{C}$ and had an emissivity value of 0.891 (a perfect black body has a maximum emissivity of 1 ). The antimicrobial effects of infrared radiation may be effective in reducing the number of 
Staphylococcus aureus and Escherichia coli bacteria by $99.9 \%$, and have shown a reduction in the viability of Klebsiella pneumoniae by 34.8\% [2-4].

\section{MATHERIALS AND METHODS}

\subsection{Functionalized textile materials}

Four functionalized textile materials (FIR + UV protection + antimicrobial functionalization compounds), figure 1 , and were subjected to antimicrobial analysis against three strains of pathogenic fungi.

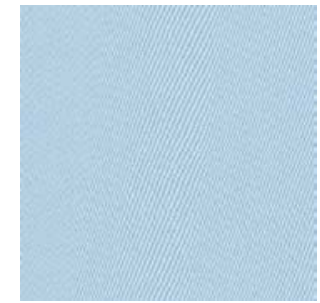

GS 102 -1

Composition: $100 \%$ PET Dying: dip dyed

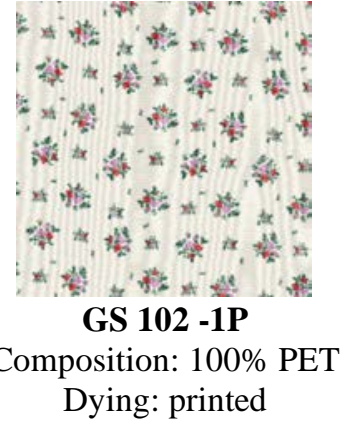

Figure 1. Functionalized textile materials

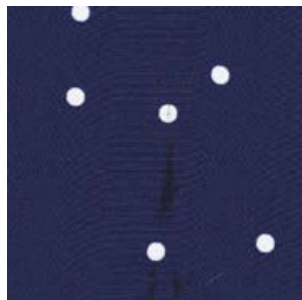

GS $102-2 P$

Composition: $100 \%$ PET

Dying: printed

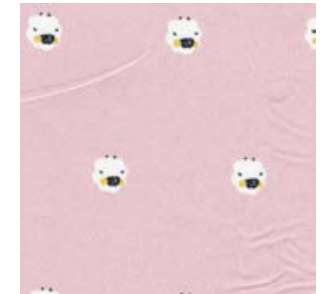

GS $102-3 P$

Composition: $75 \%$ PET + 25 BBC

Dying: printed

\subsection{Fungal strains}

The evaluation of the antimicrobial character was performed on the functionalized materials, compared with a control sample (single control, from each strain, taken as the microbial inoculum). The test was performed according to two methods, one in dynamic contact conditions (under agitation), and one in static contact conditions (inoculation on the material), and against four microbial strains of pathogenic fungi: Candida albicans (ATCC 90028), Epidermophyton floccosum (CCM 8339), Trichophyton interdigitale (ATCC 9533) and Aspergillus niger (IMI 45551).

At the end of the tests, the number of viable cells was quantified, and the degrees of microbial percentage reduction were calculated, based on the initial number of colony forming units (CFUs). In both methods, each material was tested in duplicate, and the final result was expressed as the average of the readings from two Petri plates.

Initial microbial concentrations, expressed as average of readings on two Petri plates:

Candida albicans: $3.32 \times 10^{3} \mathrm{UFC} / \mathrm{ml}$;

Epidermophyton floccosum: $3.2 \times 10^{2} \mathrm{UFC} / \mathrm{ml}$;

Tricophyton interdigitale: $4.1 \times 10^{2} \mathrm{UFC} / \mathrm{ml}$;

Aspergillus niger: $3.5 \times 10^{2} \mathrm{UFC} / \mathrm{ml}$.

\subsection{Evaluation of antimicrobial character in dynamic conditions}

Testing of the antimicrobial character of functionalized textile materials, under dynamic conditions, was performed according to the ASTM E2149 standard, which describes a standardized method for testing the antimicrobial activity of antimicrobial agents immobilized on a solid substrate under dynamic contact conditions. This method was developed for routine quality control tests and antimicrobial screening tests being used to test the character and antimicrobial efficiency of immobilized functionalization treatments on various substrates. The samples were tested according to the scheme in table 1, in 
duplicate, the results being reported by averaging the reading from two Petri plates. The quantification of the number of cells on the plates with a high number of colonies was performed with specialized software, OpenCFU, a free open-source colony counting software.

Table 1. Test scheme according to ASTM E2149

\begin{tabular}{|c|c|c|}
\hline Step & Description & Representation \\
\hline $\begin{array}{l}1 . \\
\text { Sample preparation }\end{array}$ & $\begin{array}{l}\text { Sampling, in duplicate, with a final mass of } 1 \mathrm{~g}(+/-0.1 \mathrm{~g}) \text {, and } \\
\text { placement in sterile } 100 \mathrm{ml} \mathrm{PE} \text { flasks in } 60 \mathrm{ml} \text { of sterile saline } \\
(0.875 \% \mathrm{NaCl}) \text { with Tween } 80 \text { anionic detergent }(2 \text { drops/l), in } \\
\text { order to be able to confer a better dispersion of the microbial cells } \\
\text { in the volume of liquid. Thus, the saline/Tween } 80 \text { mixture was } \\
\text { previously sterilized for } 15^{\prime} \text { at } 121^{\circ} \mathrm{C} \text {. }\end{array}$ & \\
\hline $\begin{array}{c}2 . \\
\text { Inoculation }\end{array}$ & $\begin{array}{l}\text { Stock cultures were diluted with sterile saline and Tween } 80 \\
\text { anionic detergent until final inoculation variants were obtained } \\
\text { (inoculated into sample vessels). }\end{array}$ & \\
\hline $\begin{array}{c}3 . \\
\text { Incubation }\end{array}$ & $\begin{array}{l}\text { The samples were incubated for } 24 \text { in an orbital shaking incubator } \\
\text { (SIF6000R, Medline Scientific), according to the tested strain: } \\
28^{\circ} \mathrm{C} \text { for Tricophyton interdigitale, Epidermophyton floccosum } \\
\text { and Aspergillus niger; } 36^{\circ} \mathrm{C} \text { for Candida albicans. Plates } \\
\text { inoculation was carried out in two sets, following } 1 \text { hour of } \\
\text { incubation and } 24 \text { hours of incubation. }\end{array}$ & \\
\hline $\begin{array}{l}4 . \\
\text { Sampling and plates } \\
\text { inoculation }\end{array}$ & $\begin{array}{l}\text { After the incubation period, } 100 \mu \mathrm{l} \text { of liquid were taken from each } \\
\text { sample flask and pipetted onto the Petri plates with nutrient } \\
\text { medium (Sabouraud-Agar). }\end{array}$ & \\
\hline $\begin{array}{l}5 . \\
\text { Incubation and } \\
\text { plates counting }\end{array}$ & $\begin{array}{l}\text { After plates inoculation (in duplicate) the plates were incubated at } \\
\text { a temperature of } 28^{\circ} \mathrm{C} \text { for Tricophyton interdigitale, } \\
\text { Epidermophyton floccosum and Aspergillus niger; } 36^{\circ} \mathrm{C} \text { for } \\
\text { Candida albicans, for } 48 \text { hours, timespan which allowed the } \\
\text { counting of the formed colonies. }\end{array}$ & \\
\hline
\end{tabular}

\subsection{Evaluation of antimicrobial character in static conditions}

The testing of antimicrobial efficacy, in static conditions, was done according to ISO 20743:2007, a method applicable to all textile products, including canvas, cotton wool, yarns and materials for clothing, home furniture and various products, regardless of the type of antibacterial agent used (organic, inorganic, natural or synthetic) or method of application (incorporated, post-treatment or grafting). The absorption method was used, in which the microbial suspension is inoculated directly on samples (figure 2), these being sterilized beforehand, to avoid contamination of the plates from the last stage of the protocol (1.6Plate counting). The tested materials were sampled in surfaces of $1 \mathrm{~cm}^{2}$, then sterilized at $121^{\circ} \mathrm{C}$ for $15^{\prime}$, and inoculated with $50 \mu \mathrm{L}$ of the last dilution made for each strain tested during the stage (in sterile tubes) and incubated for 24 hours at a temperature of $28^{\circ} \mathrm{C}$ for each filamentous fungal strain, and at $37^{\circ} \mathrm{C}$ for the Candida albicans strain. After the incubation period, each sample was vortexed for approx. 20" in 1mL sterile distilled water and inoculated on specific nutrient medium (Czapek-Dox and Sabouraud-Agar), followed by an incubation period of 2-3 days. The tests were performed in duplicate, for each material, and in order to quantify the results, the technique of counting the CFUs (Colony Forming Units) on the incubated plates was used, reported to the initial cell concentration in the inoculum. 

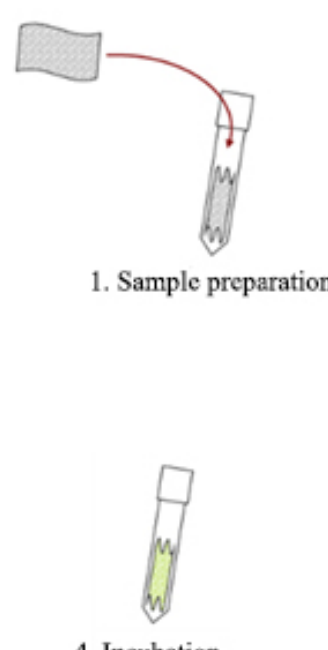

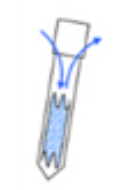

2. Sterilization

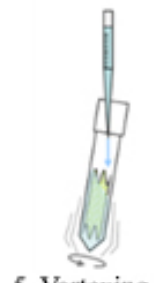

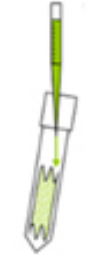

3. Inoculation

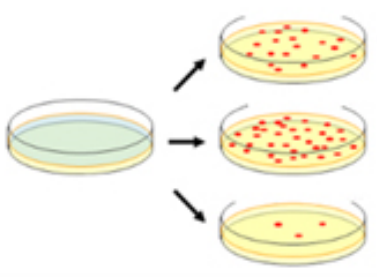

6. Plate counting

Figure 2. Test scheme according to ISO 20743:2007

\section{RESULTS AND DISCUSSION}

The results of the antimicrobial testing, on the four functionalized materials, compared to the four microbial strains, in dynamic contact conditions, are presented in table 2 and figure 3.

Table 2. Percentage microbial reductions in dynamic contact conditions

\begin{tabular}{|c|c|c|c|c|}
\hline Sample & Plate 1 & Plate 2 & Average & \% Red \\
\hline \multicolumn{5}{|c|}{ Candida albicans (3.32x10 $\left.{ }^{3} \mathrm{UFC} / \mathrm{ml}\right)$} \\
\hline GS 102-1 & $1.98 \times 10^{2}$ & $2.1 \times 10^{2}$ & $2.04 \times 10^{2}$ & 93.86 \\
\hline GS 102-1P & $1.12 \times 10^{2}$ & $1.2 \times 10^{2}$ & $1.16 \times 10^{2}$ & 96.51 \\
\hline GS 102-2P & $2.1 \times 10^{2}$ & $2.23 \times 10^{2}$ & $2.165 \times 10^{2}$ & 93.48 \\
\hline GS 102-3P & $1.78 \times 10^{2}$ & $1.65 \times 10^{2}$ & $1.715 \times 10^{2}$ & 94.84 \\
\hline \multicolumn{5}{|c|}{ Epidermophyton floccosum (3.2x10² UFC/ml) } \\
\hline GS 102-1 & $7.8 \times 10^{1}$ & $8.1 \times 10^{1}$ & $7.95 \times 10^{1}$ & 75.16 \\
\hline GS 102-1P & $6.8 \times 10^{1}$ & $6.5 \times 10^{1}$ & $6.65 \times 10^{1}$ & 79.22 \\
\hline GS 102-2P & $5.9 \times 10^{1}$ & $6.1 \times 10^{1}$ & $6.0 \times 10^{1}$ & 81.25 \\
\hline GS 102-3P & $7.1 \times 10^{1}$ & $7.3 \times 10^{1}$ & $7.2 \times 10^{1}$ & 77.50 \\
\hline \multicolumn{5}{|c|}{ Tricophyton interdigitale $\left(4.1 \times 10^{2} \mathrm{UFC} / \mathrm{ml}\right)$} \\
\hline GS 102-1 & $3.1 \times 10^{1}$ & $3.2 \times 10^{1}$ & $3.15 \times 10^{1}$ & 92.32 \\
\hline GS 102-1P & $2.8 \times 10^{1}$ & $3.0 \times 10^{1}$ & $2.9 \times 10^{1}$ & 92.93 \\
\hline GS 102-2P & $4.1 \times 10^{1}$ & $4.2 \times 10^{1}$ & $4.15 \times 10^{1}$ & 89.97 \\
\hline GS 102-3P & $3.8 \times 10^{1}$ & $3.7 \times 10^{1}$ & $3.75 \times 10^{1}$ & 90.86 \\
\hline \multicolumn{5}{|c|}{ Aspergillus niger (3.5x10² UFC/ml) } \\
\hline GS 102-1 & $5.1 \times 10^{1}$ & $5.4 \times 10^{1}$ & $5.25 \times 10^{1}$ & 85.00 \\
\hline GS 102-1P & $4.8 \times 10^{1}$ & $4.3 \times 10^{1}$ & $4.55 \times 10^{1}$ & 87.00 \\
\hline GS 102-2P & $3.9 \times 10^{1}$ & $4.2 \times 10^{1}$ & $4.05 \times 10^{1}$ & 88.43 \\
\hline GS 102-3P & $3.7 \times 10^{1}$ & $4.1 \times 10^{1}$ & $3.9 \times 10^{1}$ & 88.86 \\
\hline
\end{tabular}




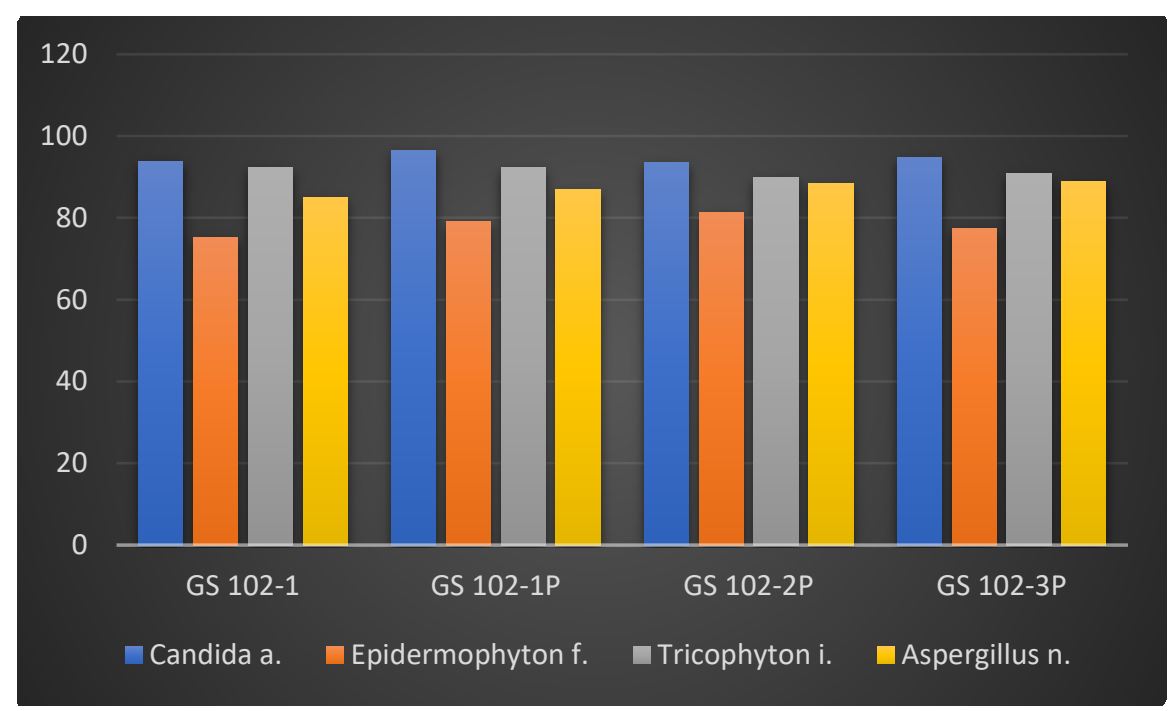

Figure 3. Comparative analysis of microbial reduction rates under dynamic conditions

The test results show very good degrees of microbial reduction, for all four materials, with values between $75.16 \%$, for the GS GS-1 material, tested against the Epidermophyton floccosum strain, and 96.51\%, for the GS 102-1P material, tested against Candida albicans strain. The materials showed the highest efficiency against Candida albicans strain, with an average microbial reduction rate of $94.67 \%$, the most resistant strain being Epidermophyton floccosum, with an average microbial reduction rate of $78.28 \%$ (table 3).

Table 3. Percentage microbial reductions in static contact conditions

\begin{tabular}{|c|c|c|c|c|}
\hline Sample & Plate 1 & Plate 2 & Average & \% Red \\
\hline \multicolumn{5}{|c|}{ Candida albicans (3.32x10 $\left.{ }^{3} \mathrm{UFC} / \mathrm{ml}\right)$} \\
\hline GS 102-1 & $2.21 \times 10^{2}$ & $2.45 \times 10^{2}$ & $2.33 \times 10^{2}$ & 92.99 \\
\hline GS 102-1P & $1.98 \times 10^{2}$ & $1.81 \times 10^{2}$ & $1.895 \times 10^{2}$ & 94.30 \\
\hline GS 102-2P & $2.45 \times 10^{2}$ & $2.56 \times 10^{2}$ & $2.505 \times 10^{2}$ & 92.46 \\
\hline GS 102-3P & $1.17 \times 10^{2}$ & $1.45 \times 10^{2}$ & $1.31 \times 10^{2}$ & 96.06 \\
\hline \multicolumn{5}{|c|}{ Epidermophyton floccosum $\left(3.2 \times 10^{2} \mathrm{UFC} / \mathrm{ml}\right)$} \\
\hline GS 102-1 & $5.1 \times 10^{1}$ & $4.9 \times 10^{1}$ & $5.0 \times 10^{1}$ & 84.38 \\
\hline GS 102-1P & $3.4 \times 10^{1}$ & $3.8 \times 10^{1}$ & $3.6 \times 10^{1}$ & 88.75 \\
\hline GS 102-2P & $2.5 \times 10^{1}$ & $2.4 \times 10^{1}$ & $2.45 \times 10^{1}$ & 92.35 \\
\hline GS 102-3P & $1.9 \times 10^{1}$ & $2.1 \times 10^{1}$ & $2.0 \times 10^{1}$ & 93.75 \\
\hline \multicolumn{5}{|c|}{ Tricophyton interdigitale $\left(4.1 \times 10^{2} \mathrm{UFC} / \mathrm{ml}\right)$} \\
\hline GS $102-1$ & $2.1 \times 10^{1}$ & $2.5 \times 10^{1}$ & $2.3 \times 10^{1}$ & 94.40 \\
\hline GS 102-1P & $4.1 \times 10^{1}$ & $3.9 \times 10^{1}$ & $4.0 \times 10^{1}$ & 90.25 \\
\hline GS 102-2P & $2.5 \times 10^{1}$ & $2.6 \times 10^{1}$ & $2.55 \times 10^{1}$ & 93.79 \\
\hline GS 102-3P & $2.3 \times 10^{1}$ & $2.1 \times 10^{1}$ & $2.2 \times 10^{1}$ & 94.94 \\
\hline \multicolumn{5}{|c|}{ Aspergillus niger $\left(3.5 \times 10^{2} \mathrm{UFC} / \mathrm{ml}\right)$} \\
\hline GS 102-1 & $2.1 \times 10^{1}$ & $2.5 \times 10^{1}$ & $2.3 \times 10^{1}$ & 93.43 \\
\hline GS 102-1P & $3.2 \times 10^{1}$ & $3.6 \times 10^{1}$ & $3.4 \times 10^{1}$ & 90.29 \\
\hline GS 102-2P & $2.8 \times 10^{1}$ & $2.7 \times 10^{1}$ & $2.75 \times 10^{1}$ & 92.15 \\
\hline GS 102-3P & $1.9 \times 10^{1}$ & $2.3 \times 10^{1}$ & $2.1 \times 10^{1}$ & 94.00 \\
\hline
\end{tabular}


The test results show very good degrees of microbial reduction, slightly better than the test results in dynamic conditions. This may be due to the test method, which allowed a longer contact time between the microbial inoculum and the functionalized material. The values of the reduction rates were between $84.38 \%$, for the GS GS-1 material, tested against Epidermophyton floccosum strain, and 96.06\%, for the GS 102-3P material, tested against the Candida albicans strain. The materials showed the highest efficiency against the Candida albicans strain, with an average microbial reduction rate of $93.95 \%$, the most resistant strain being Epidermophyton floccosum, with an average microbial reduction rate of $89.80 \%$ (figure 4 ).

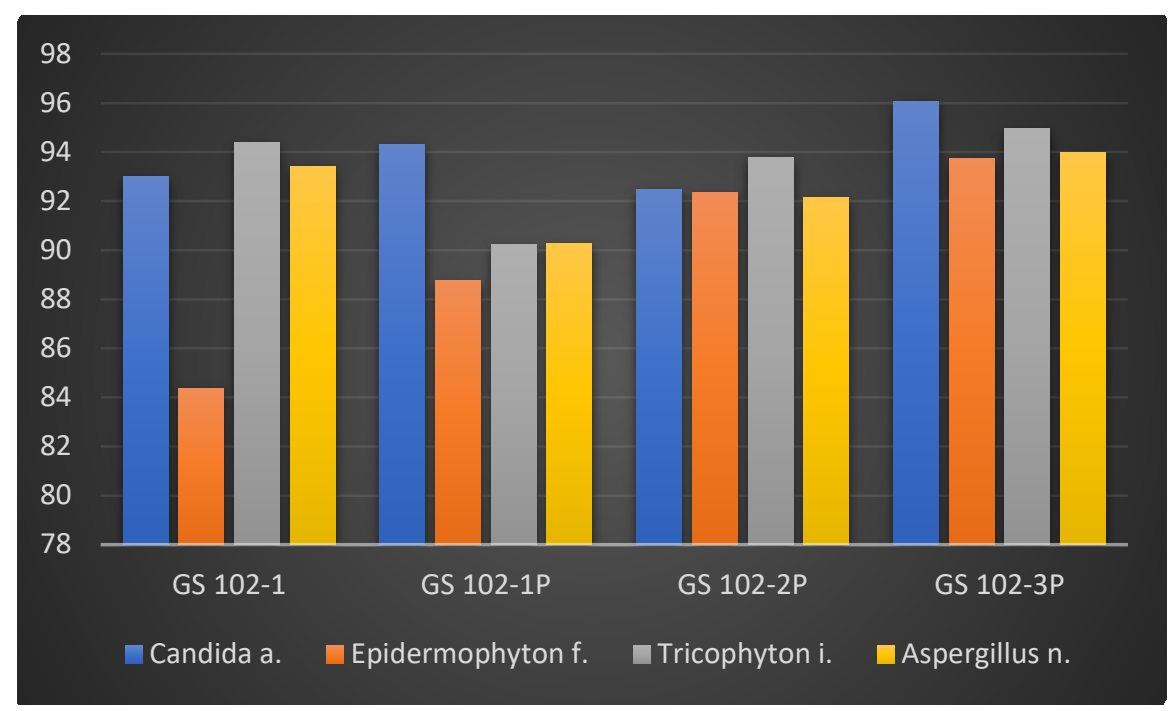

Figure 4. Comparative analysis of microbial reduction rates under static conditions

\section{CONCLUSIONS}

FIR functional textile may represent the future for alternative therapies, as they have great health benefits of FIR, ranging from regulating body heat, restoring physical function, muscle pain relieving, arthritis pain management, bronchitis etc. [7,8]. Infrared functionalized textile materials offer a unique way to apply various therapies for continuous use. These functional textile structures, existing in different forms (e.g., fibres, fabrics, composites, films etc.) have significant benefits for many types of diseases, symptoms and problems, as FIR functionality can be incorporated into textiles in a variety of ways.

The antimicrobial properties of four FIR functionalized textile materials were tested against four strains of pathogenic fungi: Candida albicans, Epydermophyton floccosum, Tricophyton interdigitale and Aspergillus niger. The evaluation of the antimicrobial character showed very good rates of reduction of the microbial population, of the functionalized textile materials, following the testing on four strains of pathogenic fungi: Candida albicans, Epydermophyton floccosum, Tricophyton interdigitale and Aspergillus niger, with reduction rates between $76.16 \%$ and $96.06 \%$.

\section{ACKNOWLEDGEMENTS}

This paper was published under Eureka Traditional program, project "Far Infrared Rays and Anion Releasing Fabrics", acronym FairTex, financed by The Executive Agency for Higher Education, Research, Development and Innovation Funding (UEFISCDI), national project ID 134E (PN-III-P3-3.5-EUK-2017-02-0044). 


\section{REFERENCES}

[1] Dyer, J., Infrared functional textiles. Functional Textiles for Improved Performance, In: Protection and Health, 2011, 184-197

[2] Toyokawa, H., et al., Promotive effects of far-infrared ray on full-thickness skin wound healing in rats, In: Exp Biol Med (Maywood), 2003, 228, 6, 724-729

[3] Chung, J., Lee, S., Development of nanofibrous membranes with far-infrared radiation and their antimicrobial properties, In: Fibers and Polymers, 2014, 15, 6, 1153-1159

[4] Tsai, S.R., Hamblin, M.R., Biological effects and medical applications of infrared radiation. Journal of photochemistry and photobiology, In: B. Biology, 2017, 170, 197-207

[5] Development of fiber-based active thermal infrared camouflage textile, In: Applied Materials Today, 2020, 20, 100624

[6] Ying-Nan, S., Yue, L., Ding-Xiang, Y., Jun, L., Zhong-Ming, L., Novel passive cooling composite textile for both outdoor and indoor personal thermal management, In: Composites Part A: Applied Science and Manufacturing, 2020, 130, 105738 